\title{
LPS Up-Regulates ICAM-1 Expression in Breast Cancer Cells by Stimulating a MyD88-BLT2-ERK- Linked Cascade, Which Promotes Adhesion to Monocytes
}

\author{
Geun-Soo Park, and Jae-Hong Kim*
}

\begin{abstract}
Monocytes are the major inflammatory cells that infiltrate most solid tumors in humans. The interaction of tumor cells with infiltrating monocytes and their adhesion to these monocytes play a significant role in altering the tumor to become more aggressive. Recently, exposure to lipopolysaccharide (LPS) was suggested to promote cancer cell adhesion to monocytes; however, little is known about the details of the signaling mechanism involved in this process. In this study, we found that LPS up-regulates ICAM-1 expression in MDA-MB-231 breast cancer cells, which facilitates their adhesion to THP-1 monocytes. In addition, we analyzed the signaling mechanism underlying the up-regulation of ICAM-1 and found that the SiRNAmediated depletion of BLT2 markedly suppressed the LPSinduced expression of ICAM-1 in MDA-MB-231 cells and the subsequent adhesion of these cells to THP-1 monocytes. Moreover, we demonstrated that myeloid differentiation primary response gene 88 (MyD88) lies downstream of LPS/TLR4 and upstream of BLT2 and that this 'MyD88BLT2' cascade mediates ERK activation and subsequent ICAM-1 expression, which is critical for the adhesion of MDA-MB-231 cells to THP-1 monocytes. Taken together, our results demonstrate for the first time that LPS upregulates ICAM-1 expression in breast cancer cells via a MyD88-BLT2-ERK-linked signaling cascade, leading to the increased adhesion of breast cancer cells to monocytes.
\end{abstract}

\section{INTRODUCTION}

Tumor progression proceeds through a series of complex events, each of which is accelerated by stromal cells, including fibroblasts, immune cells, endothelial cells and pericytes, in the tumor microenvironment (Mantovani et al., 2008). In particular, monocytes are actively recruited to tumors, which

School of Life Sciences and Biotechnology, Korea University, Seoul 136701 , Korea

*Correspondence: jhongkim@korea.ac.kr

Revised 16 June, 2015; revised 17 July, 2015; accepted 17 July, 2015; published online 21 August, 2015

Keywords: beast cancer, BLT2, ICAM-1, LPS, MyD88 they then infiltrate, and the direct contact between cancer cells and monocytes has been suggested to be a critical component of tumor progression (Blot et al., 2003; Chittezhath et al., 2014). Although recent reports have suggested that lipopolysaccharide (LPS)/Toll-like receptor 4 (TLR4) signaling stimulates the adhesion of cancer cells to monocytes or endothelial cells, the detailed mechanism by which LPS promotes the adhesion of cancer cells to these cells remain unknown (Chen and Khismatullin, 2014; Kim et al., 2013b; Lee et al., 2013).

Intercellular adhesion molecule (ICAM)- 1 is a member of the immunoglobulin superfamily and is expressed on many cell types, including endothelial cells, leukocytes and cancer cells, where it can be upregulated in response to various proinflammatory cytokines or stimuli (Hubbard and Rothlein, 2000). ICAM-1 expression in cancer cells has been correlated with progression to a more aggressive state and has been shown to mediate various cancer-related biological processes such as survival, migration, extravasation, homing and metastasis (Evani et al., 2013; Huang et al., 2004; Rosette et al., 2005; Schroder et al., 2011; Zhu and Gong, 2013). ICAM-1 expressed on cancer cells binds to integrins belonging to the $\beta 2$ subfamily, such as CD11b/CD18 (Mac-1), on the surface of monocytes (van de Stolpe and van der Saag, 1996). However, the signaling pathway that results in ICAM-1 up-regulation in cancer cells, particularly in response to LPS, is not fully understood.

Leukotriene $B_{4}\left(L T B_{4}\right)$ is a potent chemoattractant and a local pro-inflammatory lipid mediator that plays a role in innate immunity (Funk, 2001). Recent studies have suggested that $\mathrm{LTB}_{4}$ and its receptor BLT2 are associated with aspects of cancer progression including invasion, epithelialmesenchymal transition (EMT) and metastasis (Kim et al., 2012; 2014; Seo et al., 2012). In addition, LPS treatment increases the production of BLT2 ligands such as $\mathrm{LTB}_{4}$ and 12(S)-hydroxyeicosatetraenoic acid (12(S)-HETE) in MDA-MB231 cells, but the exact role of the BLT2 ligand-BLT2 axis remains to be determined, particularly regarding adhesion to monocytes (Park and Kim, 2015).

In this study, we found that LPS enhances the adhesion of MDA-MB-231 breast cancer cells to THP-1 monocytes and that one of the mechanisms by which LPS promotes this adhesion involves the upregulation of ICAM-1. Moreover, we found that this LPS-induced expression of ICAM-1 in MDA-MB-231 cells 
was markedly suppressed by the siRNA-mediated depletion of BLT2. In addition, we showed that MyD88 functions upstream of BLT2 and ERK functions downstream. Together, our results describe a novel LPS-induced MyD88-BLT2-ERK-ICAM-1 signaling cascade in MDA-MB-231 cells that promotes their adhesion to monocytes. Thus, our findings provide novel insight into how LPS increases the adhesion of cancer cells to monocytes, a process that is critical for cancer progression.

\section{MATERIALS AND METHODS}

\section{Materials}

Fetal bovine serum (FBS) and RPMI 1640 were obtained from Life Technologies (USA). MK886, Baicalein and LY255283 were purchased from Cayman Chemical (USA). LPS (Escherichia coli serotype O55:B5), bovine serum albumin, and dimethyl sulfoxide (DMSO) were purchased from Sigma-Aldrich (USA). PD98059 was purchased from Calbiochem (USA). An antibody to ICAM-1 was obtained from Santa Cruz Biotechnology (USA), and antibodies against phospho-ERK (p-ERK), ERK and $\alpha$-tubulin were obtained from Cell Signaling Technology (USA). R-phycoerythrin (PE)-conjugated mouse antihuman Mac-1 antibody, PE-conjugated mouse anti-human ICAM-1 antibody and PE-conjugated mouse IgG isotype control antibody were obtained from BD Biosciences (USA). All other chemicals were obtained from standard sources and were of molecular biology grade or higher.

\section{Cell culture}

The human breast cancer cell line MDA-MB-231 and the human monocyte cell line THP-1 were obtained from the Korean Cell Line Bank (Korea). MDA-MB-231 cells were maintained in RPMI-1640 containing 10\% heat-inactivated FBS and antibiotic-antimycotic solution (Life Technologies) at $37^{\circ} \mathrm{C}$ in a humidified atmosphere of $5 \% \mathrm{CO}_{2}$. THP-1 cells were maintained in RPMI-1640 containing 10\% heat-inactivated FBS, antibioticantimycotic solution and $0.5 \mathrm{mM}$ mercaptoethanol at $37^{\circ} \mathrm{C}$ in a humidified atmosphere of $5 \% \mathrm{CO}_{2}$.

\section{Semi-quantitative RT-PCR}

Total RNA was extracted from cells with the use of Easy Blue (Intron, Korea). Aliquots $(1.25 \mu \mathrm{g})$ of the RNA were subjected to RT with M-MLV reverse transcriptase (Beams Bio, Korea) followed by semi-quantitative PCR analysis with a PCR PreMix Kit (Intron) under conditions optimal for the linear amplification of Glyceraldehyde 3-phosphate dehydrogenase (GAPDH) cDNA. The primer sequences used are as follows: human ICAM-1 (forward, 5'-CCGGAAGGTGTATGAACTG-3'; reverse, 5'-TCCATGGTGATCTCTCCTC-3') (Silverman et al., 2001); human BLT2 (forward, 5'- AGCCTGGAGACTCTGACCGC TTTCG-3'; reverse, 5'-GACGTAGCACCGGGTTGACGCTA-3') (Seo et al., 2011); human MyD88 (forward, 5'-TCTCTGTTCTT GAACGTGCGGACA-3'; reverse, 5'-TTTGGCAATCCTCCTCA ATGCTGG-3'); and GAPDH (forward, 5'-CTGCACCACCAACT GCTTAGC-3'; reverse, 5'-CTTCACCACCTTCTTGATGTC-3') (Seo et al., 2011). The specificity of all primers was confirmed by sequencing the PCR products.

\section{Western blot analysis}

The cells were washed with ice-cold PBS, scraped into lysis buffer (20 mM Tris- $\mathrm{HCl}$, pH 7.5, $150 \mathrm{mM} \mathrm{NaCl}, 0.5 \%$ Nonidet $\mathrm{P}$ 40, 5 mM EDTA, 1\% Triton X-100, and protease inhibitors (100 $\mathrm{mM}$ phenylmethylsulfonyl fluoride, $1 \mathrm{mM}$ sodium orthovanadate, $2 \mu \mathrm{g} / \mathrm{ml}$ leupeptin, and $2 \mu \mathrm{g} / \mathrm{ml}$ aprotinin) at $4^{\circ} \mathrm{C}$, and heated at $95^{\circ} \mathrm{C}$ for $5 \mathrm{~min}$. The samples were then subjected to SDSPAGE, and the separated proteins were transferred electrophoretically to a PVDF membrane for $90 \mathrm{~min}$ at $100 \mathrm{~V}$. After exposure for $1 \mathrm{~h}$ to TBST containing $0.05 \%$ Tween 20 and $5 \%$ dried nonfat milk, the membranes were incubated overnight at $4^{\circ} \mathrm{C}$ with antibodies specific for ICAM-1, p-ERK, ERK or $\alpha$-tubulin (loading control). The blots were developed with peroxidaseconjugated secondary antibodies, and the proteins were visualized using ECL reagents (Amersham, USA) according to the manufacturer's recommendations.

\section{Adhesion assay}

THP-1 cells were labeled with $10 \mu \mathrm{M}$ calcein-AM (Molecular Probes, USA) for $1 \mathrm{~h}$ at $37^{\circ} \mathrm{C}$. LPS-treated MDA-MB-231 cells were washed with $\mathrm{PBS}$, and THP-1 cells were seeded onto the MDA-MB-231 cells. After $1 \mathrm{~h}$, these co-cultured cells were washed with PBS, and the adhesion of THP-1 cells to MDAMB-231 cells was observed under a fluorescence microscope (Axiovert 200, Carl Zeiss, Germany) and quantified.

\section{RNA interference (RNAi)}

BLT2-specific (5'-CCACGCAGUCAACCUUCUG-3')(Chaves et al., 2014), MyD88-specific (5'-AGUAGAGCACAGAUUC CUC-3'; No. 1100256) and control (scrambled) siRNAs were obtained from Bioneer (Korea). The siRNAs were introduced into the cells by transfection for the indicated times in Opti-MEM solution (Invitrogen, USA) using Oligofectamine reagents (Invitrogen).

\section{Forced expression of BLT2 and MyD88}

Cells were transiently transfected with $1 \mu \mathrm{g}$ of the expression vector for human BLT2 (pcDNA3.1-BLT2) (Kim et al., 2010) and MyD88 (pCMV-Flag-MyD88) (Coste et al., 2010) or the corresponding empty vectors (pcDNA3.1 and pCMV-Flag) using Lipofectamine reagents (Invitrogen).

\section{ELISAS}

ELISA kits for human $\mathrm{LTB}_{4}$ and 12(S)-HETE were obtained from Enzo life sciences (USA). The $\mathrm{LTB}_{4}$ and 12(S)-HETE concentrations were measured according to the manufacturer's instructions.

\section{Data analysis and statistics}

The data are representative of three independent experiments. The results are presented as means \pm standard deviation (SD). Analyses were performed with Student's $t$-test using SigmaPlot 8.0 software. $P$-Values of less than 0.05 were considered significant.

\section{RESULTS}

ICAM-1 expression induced by LPS in MDA-MB-231 breast cancer cells promotes their adhesion to THP-1 monocytes We assessed whether LPS enhanced the adhesion of MDAMB-231 cells to THP-1 monocytes and found that adhesion was significantly increased by LPS treatment (Fig. 1A). To understand the mechanism by which LPS enhances the adhesion of MDA-MB-231 cells to monocytes, we examined whether LPS treatment up-regulates ICAM-1 expression in MDA-MB231 cells. Semi-quantitative RT-PCR and western blot analyses revealed that the levels of ICAM-1 mRNA and protein, respectively, were markedly increased by LPS (Fig. 1B). ICAM-1 has been reported to directly interact with Mac-1 on the surface of monocytes (Usami et al., 2013). Therefore, to examine whether 
$\boldsymbol{A}$
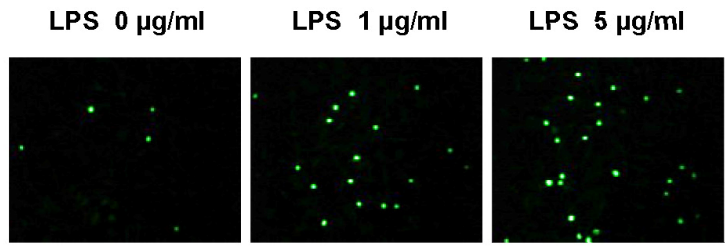

$\boldsymbol{B}$

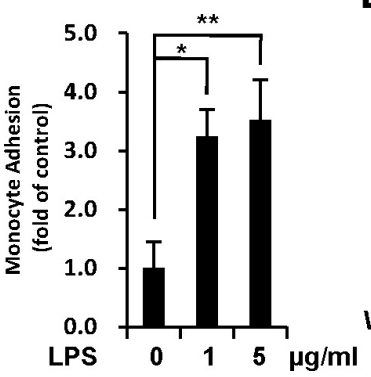

C
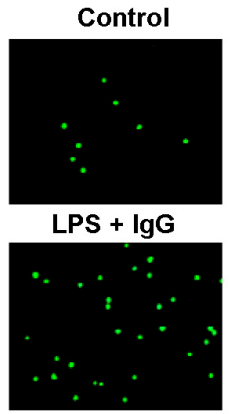
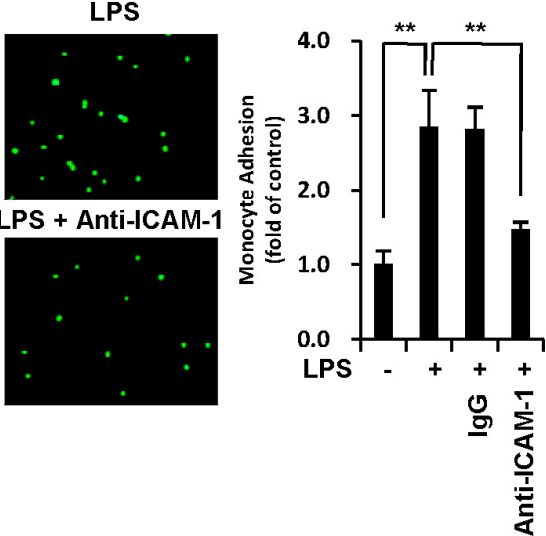

D
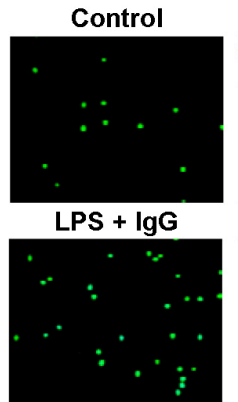
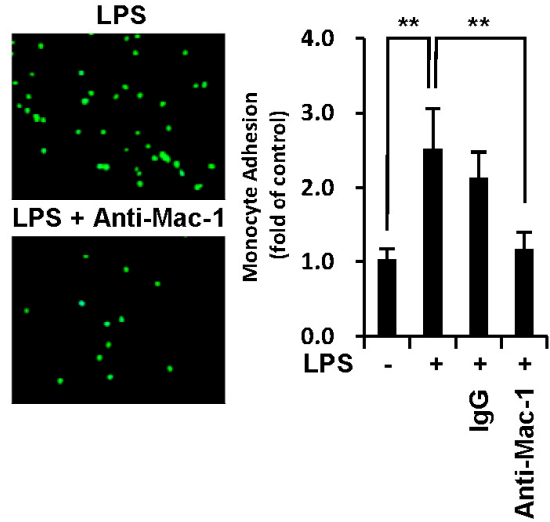

Fig. 1. ICAM-1 expression induced by LPS in MDA-MB-231 breast cancer cells promotes their adhesion to THP-1 monocytes. (A) MDA-MB231 cells were treated with LPS $(0,1$, and $5 \mu \mathrm{g} / \mathrm{ml})$ for $24 \mathrm{~h}$ and then co-cultured with calcein-AM-labeled THP-1 cells for $1 \mathrm{~h}$. THP-1 cells that had adhered to MDA-MB-231 cells were visualized, and the number of adherent monocytes was determined using fluorescence microscopy. (B) MDA-MB-231 cells were treated with LPS $(0,1$, and $5 \mu \mathrm{g} / \mathrm{ml})$ for $24 \mathrm{~h}$, after which the mRNA levels of ICAM-1, BLT2, MyD88 and GAPDH were measured by semi-quantitative RT-PCR. The protein levels of ICAM-1 and $\alpha$-tubulin were measured by Western blot assay. (C) LPSpretreated MDA-MB-231 cells $(1 \mu \mathrm{g} / \mathrm{ml}, 24 \mathrm{~h})$ were treated with anti-human ICAM-1 or anti-mouse IgG isotype control antibodies for $2 \mathrm{~h}$, and the cells were then co-cultured with calcein-AM-labeled THP- 1 cells for $1 \mathrm{~h}$. The adherence of THP-1 cells to MDA-MB-231 cells was visualized, and the number of adherent monocytes was determined using fluorescence microscopy. (D) THP-1 cells were treated with anti-human Mac-1 or anti-mouse IgG isotype control antibodies for $2 \mathrm{~h}$, and the THP-1 cells with calcein-AM-label were then co-cultured with LPSpretreated MDA-MB-231 cells for $1 \mathrm{~h}$. The adherence of THP-1 cells to MDA-MB-231 cells was visualized, and the number of adherent monocytes was determined using fluorescence microscopy. The data are representative of three independent experiments with similar results. All quantitative data are represented as the mean \pm SD from three independent experiments. ${ }^{*} p<0.05,{ }^{* *} p<0.01$.

the adhesion of MDA-MB-231 cells to monocytes is mediated through interactions between ICAM-1 on the MDA-MB-231 cells and Mac- 1 on the THP-1 monocytes, the cells were pretreated with anti-human ICAM-1 antibody (for MDA-MB-231 cells) or anti-human Mac-1 antibody (for THP-1 cells). The adhesion of THP-1 monocytes to LPS-pretreated MDA-MB-231 cells was significantly inhibited by either anti-ICAM-1 or antiMac-1 antibody pretreatment (Figs. 1C and 1D). Together, these results suggest that LPS-induced ICAM-1 expressed on MDA-MB-231 cells interacts with Mac-1 on monocytes.

\section{BLT2 inhibition attenuates LPS-induced ICAM-1 expres- sion in MDA-MB-231 cells}

Interestingly, we found that the level of BLT2 expression was also significantly elevated in MDA-MB-231 cells following LPS treatment (Fig. 1B). Therefore, we next investigated whether the up-regulated BLT2 plays a role in the expression of ICAM-
1 in MDA-MB-231 cells, thereby mediating their adhesion to THP-1 monocytes. To test this hypothesis, we examined the effects of BLT2 depletion and found that LPS-induced ICAM-1 expression was clearly suppressed by the siRNA-mediated knockdown of BLT2 in MDA-MB-231 cells (Fig. 2A). In addition, BLT2 knockdown clearly abolished the adhesion of MDA-MB-231 cells to THP-1 monocytes (Fig. 2B). Furthermore, we found that the overexpression of BLT2 alone results in the upregulation of ICAM-1 at both the transcript and protein levels (Fig. 2C). Together, these results suggest that the up-regulated BLT2 plays a role in the expression of ICAM- 1 in MDA-MB-231 cells, thereby mediating their adhesion to THP1 monocytes.

Inhibition of BLT2 ligands synthesis suppresses LPSinduced ICAM-1 expression in MDA-MB-231 cells

The biosynthesis of the BLT2 ligands, $\mathrm{LTB}_{4}$ and 12(S)-HETE, 
$\boldsymbol{A}$

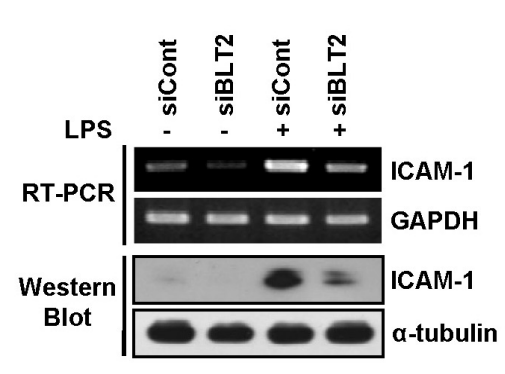

B

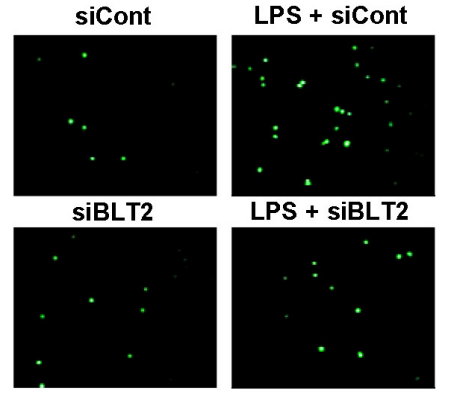

C

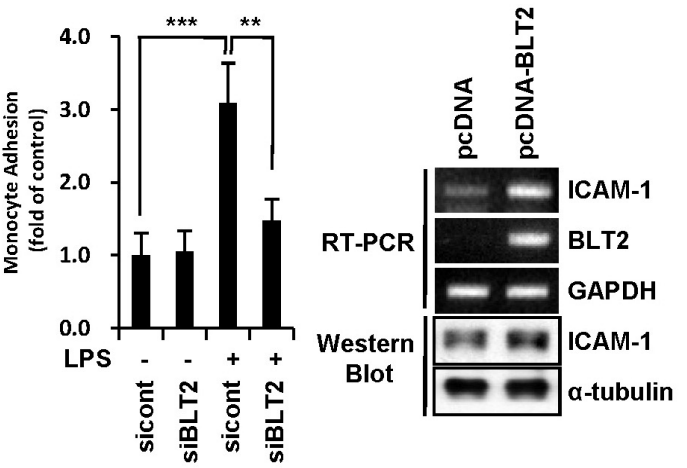

Fig. 2. BLT2 inhibition attenuates LPS-induced ICAM-1 expression in MDA-MB-231 cells. (A) MDA-MB-231 cells were transfected with BLT2 (siBLT2) or control (siCont) siRNA for $24 \mathrm{~h}$ and then treated with LPS $(1 \mu \mathrm{g} / \mathrm{ml})$ for $24 \mathrm{~h}$. The ICAM-1 mRNA levels in these treated MDA-MB231 cells were assayed by semi-quantitative RT-PCR (upper), and the ICAM-1 protein levels were determined by Western blot assay (lower). (B) MDA-MB-231 cells were transfected and treated as in (A) and then co-cultured with calcein-AM-labeled THP-1 cells for $1 \mathrm{~h}$. The adherence of THP-1 cells to MDA-MB-231 cells was then visualized, and the number of adherent monocytes was determined using fluorescence microscopy. (C) MDA-MB-231 cells were transfected with an expression plasmid for human BLT2 (pcDNA-BLT2) or the empty plasmid (pcDNA), and then incubated for $24 \mathrm{~h}$. Then, the cells were assayed for ICAM-1 mRNA levels by semiquantitative RT-PCR (upper) and protein levels by Western blot assay (lower). The data are representative of three independent experiments with similar results. All quantitative data are represented as the mean $\pm \mathrm{SD}$ from three independent experiments. ${ }^{* *} p<0.01,{ }^{* \star *} p<0.005$.

$\boldsymbol{A}$
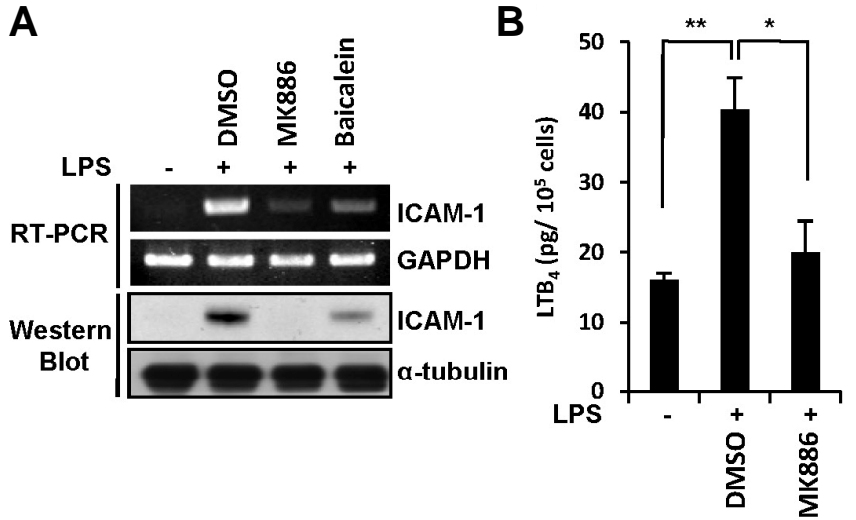

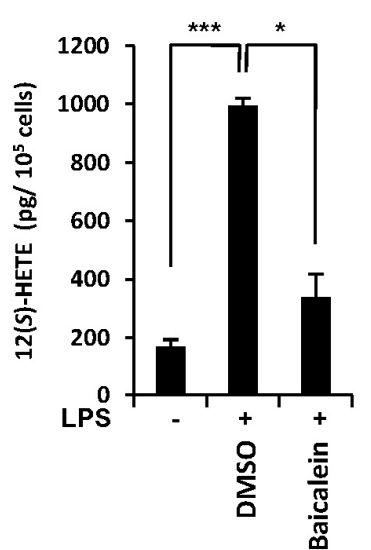

C

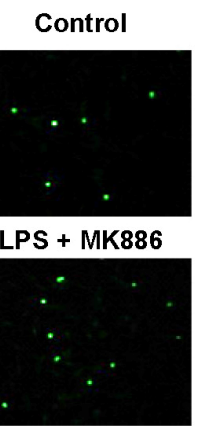

LPS

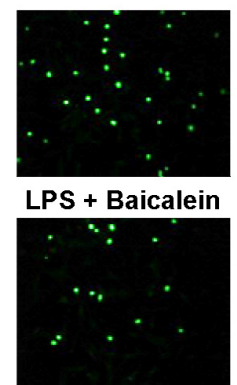

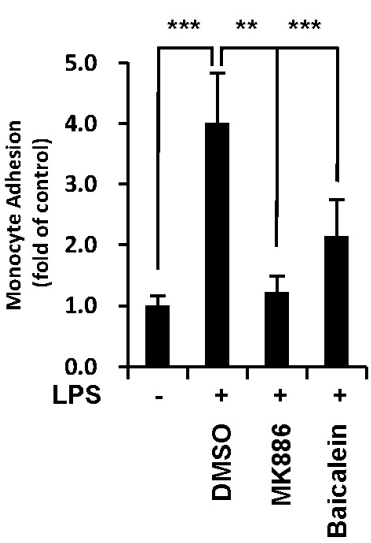

Fig. 3. Inhibition of BLT2 ligands synthesis suppresses LPSinduced ICAM-1 expression in MDA-MB-231 cells. (A) MDAMB-231 cells were incubated with MK886 $(5 \mu \mathrm{M})$ and baicalein $(20 \mu \mathrm{M})$ for $30 \mathrm{~min}$ and then incubated for $24 \mathrm{~h}$ in the presence or absence of LPS (1 $\mu \mathrm{g} / \mathrm{ml})$. The cells were then assayed for ICAM-1 mRNA levels by semi-quantitative RTPCR (upper) and protein levels by Western blot assay (lower). (B) MDA-MB-231 cells were treated as in $(A)$ and then the levels of $\mathrm{LTB}_{4}$ and 12(S)-HETE in cell supernatants were measured by ELISA. (C) MDA-MB231 cells were treated as in (A) and then co-cultured with calcein-AM-labeled THP-1 cells for 1 h. The adherence of THP-1 cells to MDA-MB-231 cells was visualized, and the number of adherent monocytes was determined using fluorescence microscopy. The data are representative of three independent experiments with similar results. All quantitative data are represented as the mean \pm SD from three independent experiments. ${ }^{*} p<0.05,{ }^{* *} p$ $<0.01,{ }^{* *} p<0.005$. 
$\boldsymbol{A}$

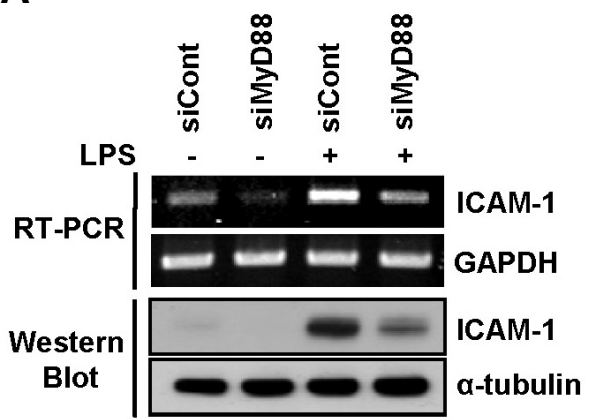

B

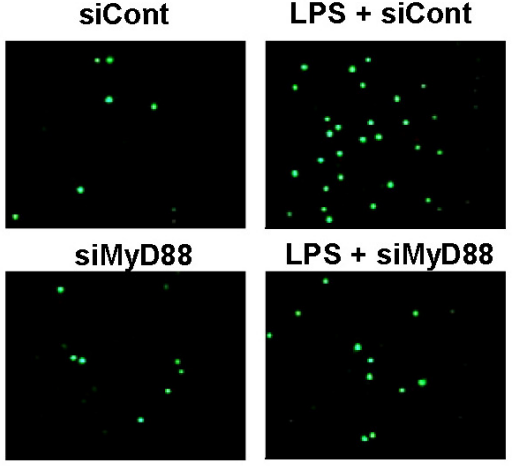

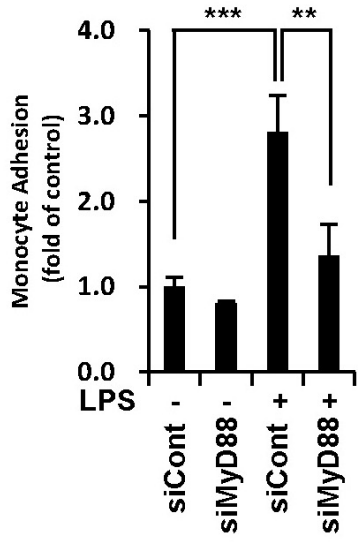

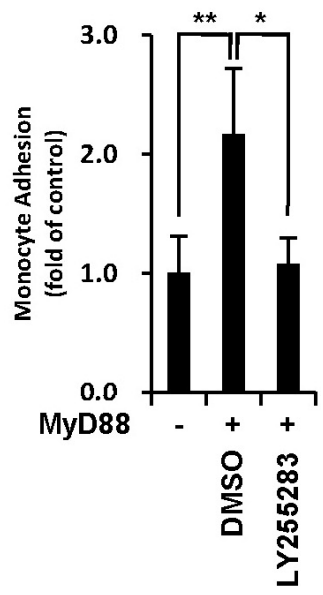

C

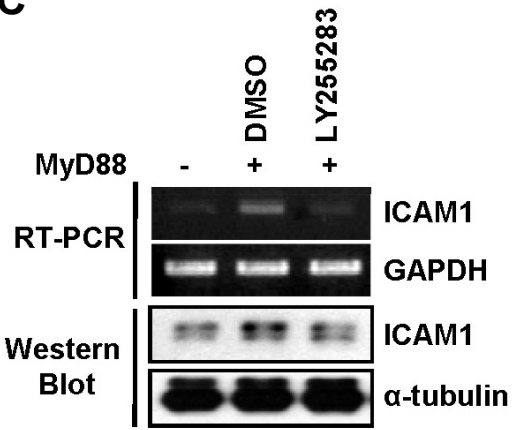

D

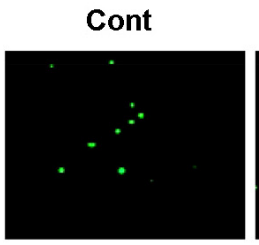

MyD88

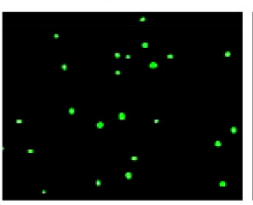

MyD88 + LY255283

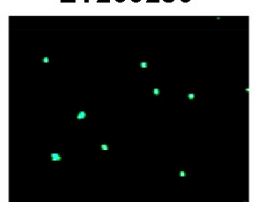

Fig. 4. A MyD88-BLT2 cascade mediates the induction of ICAM-1 expression in MDA-MB-231 cells by LPS. (A) MDA-MB-231 cells were transfected with MyD88 (siMyD88) or control (siCont) siRNA for $24 \mathrm{~h}$ and then treated with LPS $(1 \mu \mathrm{g} / \mathrm{ml})$ for $24 \mathrm{~h}$. The ICAM-1 mRNA and protein levels within the cells were assayed by semi-quantitative RT-PCR (upper) and Western blot assay (lower), respectively. (B) MDA-MB231 cells were transfected and treated as in (A) and then co-cultured with calcein-AM-labeled THP-1 cells for $1 \mathrm{~h}$. The adherence of THP-1 cells to MDA-MB-231 cells was visualized, and the number of adherent monocytes was determined using fluorescence microscopy. (C) MDAMB-231 cells were transfected with an expression plasmid for MyD88 or an empty plasmid (Cont) and then incubated for $24 \mathrm{~h}$. MDA-MB-231 cells were treated with the BLT2 inhibitor LY255823 for $24 \mathrm{~h}$ and then assayed for ICAM-1 mRNA levels by semi-quantitative RT-PCR (upper) and ICAM-1 protein levels by Western blot (lower). (D) MDA-MB-231 cells were transfected and treated as in (C) and then co-cultured with calcein-AM-labeled THP-1 cells for $1 \mathrm{~h}$. The adherence of THP-1 cells to MDA-MB-231 cells was visualized, and the number of adherent monocytes was determined using fluorescence microscopy. The data are representative of three independent experiments with similar results. All quantitative data are represented as the mean $\pm \mathrm{SD}$ from three independent experiments. ${ }^{*} p<0.05,{ }^{* *} p<0.01,{ }^{* * *} p<0.005$.

from arachidonic acid is catalyzed by 5-lipoxygenase (5-LO) and 12-lipoxygenase (12-LO), respectively (Powell et al., 1999). Previously, we reported that the levels of $\mathrm{LTB}_{4}$ and $12(\mathrm{~S})$-HETE were significantly increased in MDA-MB-231 cells by treatment with LPS (Park and Kim, 2015). To further demonstrate the role of BLT2 in the expression of ICAM-1, we pretreated the cells with the 5-LO activating protein (FLAP) inhibitor MK886 or 12-LO inhibitor baicalein and found that both ICAM-1 levels and adhesion to THP-1 cells were markedly suppressed in LPS-treated MDA-MB-231 cells (Figs. 3A and 3C). Under these experimental conditions, the LPS-induced increases in the levels of BLT2 ligands $\left[\mathrm{LTB}_{4}\right.$ and $12(\mathrm{~S})$-HETE] were clearly suppressed by MK886 or baicalein, respectively (Fig. 3B). Together, these results suggest that BLT2 ligands [ $\mathrm{LTB}_{4}$ and $\left.12(\mathrm{~S})-\mathrm{HETE}\right]$ are ne- cessary for the LPS-enhanced ICAM-1 expression.

\section{A MyD88-BLT2 cascade mediates LPS-induced ICAM-1 expression in MDA-MB-231 cells}

Previously, we suggested that MyD88 acts downstream of LPS/TLR4 and upstream of BLT2 (Park and Kim, 2015). Therefore, we next tested whether MyD88 acts upstream of BLT2 in the LPS signaling pathway to stimulate ICAM-1 expression in MDA-MB-231 cells. The siRNA-mediated depletion of MyD88 in LPS-treated MDA-MB-231 cells resulted in a marked attenuation of ICAM-1 expression (Fig. 4A) and adhesion to THP-1 monocytes (Fig. 4B). Consistent with these results, we found that the overexpression of MyD88 alone results in the upregulation of ICAM-1 at both the transcript and protein levels whereas 
A

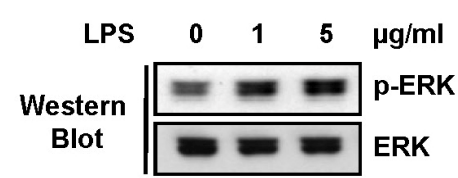

$C$

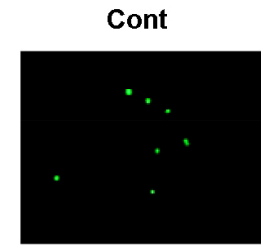

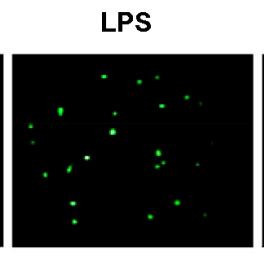

B

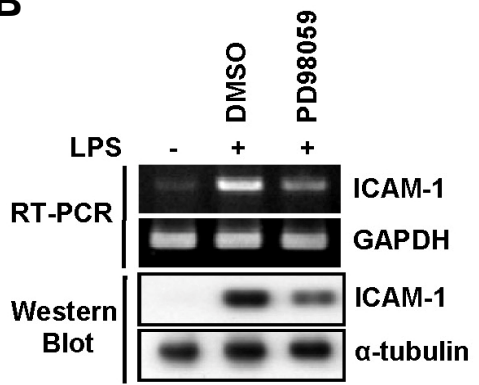

$\boldsymbol{E}$

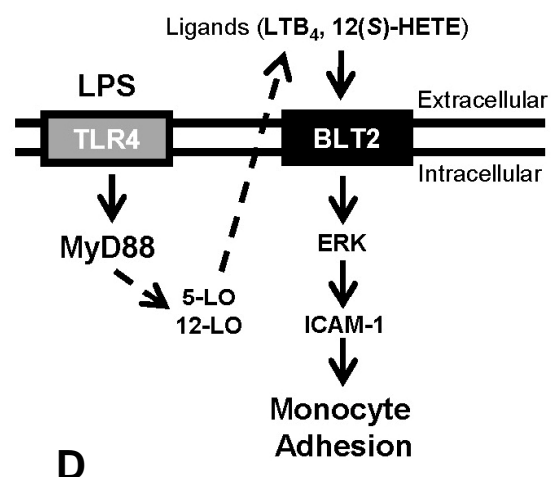

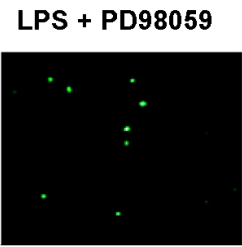
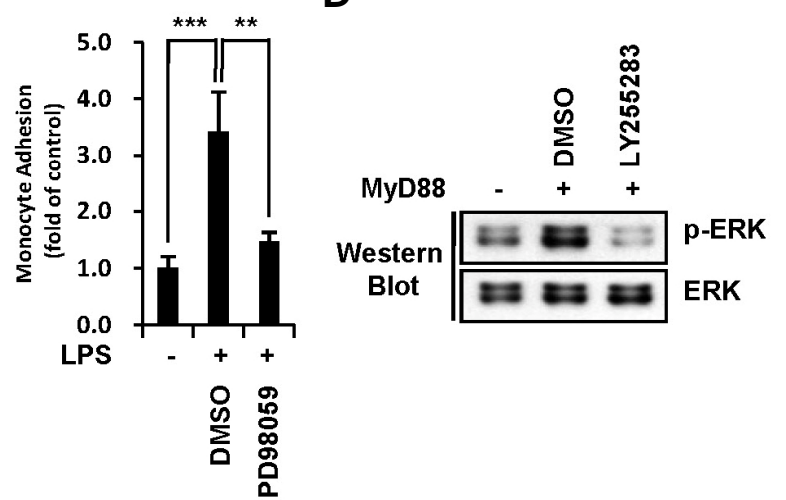

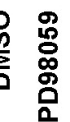

Fig. 5. ERK lies downstream of BLT2 in induction of ICAM-1 expression by LPS. (A) MDA-MB- 231 cells were treated with LPS (0, 1 , and 5 $\mu \mathrm{g} / \mathrm{ml}$ ) for $24 \mathrm{~h}$, and then the levels of $\mathrm{p}$-ERK and ERK were measured by Western blotting. (B) MDA-MB-231 cells were incubated for 30 min with PD98059 $(10 \mu \mathrm{M})$ and then incubated for $24 \mathrm{~h}$ in the presence or absence of LPS $(1 \mu \mathrm{g} / \mathrm{ml})$. The cells were assayed for ICAM-1 mRNA levels by semi-quantitative RT-PCR (upper) and for ICAM-1 protein levels by Western blotting (lower). (C) MDA-MB-231 cells were treated as in (B) and then co-cultured with calcein-AM-labeled THP-1 cells for $1 \mathrm{~h}$. The adherence of THP-1 cells to MDA-MB-231 cells was visualized, and the number of adherent monocytes was determined using fluorescence microscopy. (D) MDA-MB-231 cells were transfected with an expression plasmid for MyD88 or an empty plasmid and then treated with LY255283 for $24 \mathrm{~h}$. The cells were assayed for $\mathrm{p}$-ERK and ERK protein levels by Western blot. (E) The scheme for the involvement of the MyD88-BLT2-ERK cascade in the induction of ICAM-1 expression in MDA-MB-231 cells by LPS and the subsequent adhesion of those cells to monocytes. The data are representative of three independent experiments with similar results. All quantitative data are represented as the mean \pm SD from three independent experiments. ${ }^{\star \star} p<0.01$, ${ }^{\star \star \star} p<$ 0.005 .

BLT2 inhibition by LY255283 abolishes the expression of ICAM1 (Fig. 4C). In addition, the inhibition of BLT2 in MyD88transfected MDA-MB-231 cells leads to a clear reduction in their adhesion to THP-1 monocytes (Fig. 4D). Together, these results suggest that LPS induces ICAM-1 expression in MDAMB-231 cells through a MyD88-BLT2-linked cascade.

\section{ERK lies downstream of BLT2 in induction of ICAM-1 expression by LPS}

To understand the signaling downstream of BLT2 that is involved in the LPS-induced expression of ICAM-1, we examined the role of ERK. ERK phosphorylation is increased following exposure to LPS (Fig. 5A). We also observed that the pretreatment of MDA-MB-231 cells with PD98059, an ERK inhibitor, significantly suppressed both the LPS-mediated induction of ICAM-1 expression at the transcript and protein levels (Fig. 5B) and the subsequent adhesion to THP-1 monocytes (Fig. 5C). However, pretreatment with C-Jun $\mathrm{N}$-terminal kinase inhibitor SP600125 or p38 kinase inhibitor SB203580 had no effect on
LPS-enhanced ICAM-1 expression (data not shown). To investigate whether ERK activation lies downstream of the MyD88BLT2 cascade, we examined the effect of BLT2 inhibition on the phosphorylation of ERK in MyD88-overexpressing MDA-MB231 cells and found that MyD88-induced ERK phosphorylation was significantly suppressed by the inhibition of BLT2 by LY255283 (Fig. 5D). These data suggest that ERK lies downstream of the MyD88-BLT2 cascade involved in the LPSmediated stimulation of ICAM-1 expression in MDA-MB-231 cells.

\section{DISCUSSION}

In the present study, we found that LPS stimulates ICAM-1 expression in MDA-MB-231 cells via a BLT2-linked signaling cascade, which in turn facilitates the adhesion of these cells to THP-1 monocytes. In addition, we identified that MyD88, a pivotal regulator of the TLR4 signaling pathway, acted upstream of BLT2 and that ERK acted downstream of BLT2, mediating 
the LPS-induced expression of ICAM-1. Together, our findings show that the treatment of breast cancer cells with LPS enhances their expression of ICAM-1 through a MyD88-BLT2ERK signaling cascade, which leads to breast cancer cell adhesion to monocytes.

LPS is a key component of the outer membrane of Gramnegative bacteria and is specifically recognized by TLR4. LPSmediated signaling has been shown to play an important role in regulating cancer progression (Rakoff-Nahoum and Medzhitov, 2009). However, its role in regulating ICAM-1 in cancer cells and their adhesion to monocytes remains unknown. In the present study, we found that LPS up-regulates ICAM-1 expression via a BLT2-dependent pathway in MDA-MB-231 cells (Fig. $2 A$ ), thus facilitating the adhesion of MDA-MB-231 cells to THP1 monocytes (Fig. 2B). BLT2 is a G-protein-coupled receptor that is expressed on the cell surface and interacts with the specific ligands $\mathrm{LTB}_{4}$ and $12(\mathrm{~S})$-HETE. Previous studies have suggested that BLT2 plays roles in breast cancer cell invasion, metastasis, and chemoresistance (Kim et al., 2012; 2013a; Park and Kim, 2015). However, the upstream regulators of BLT2 in breast cancer cells remain to be identified, and the 'LPS-MyD88' cascade was only recently shown to act upstream of BLT2, thus potentiating the invasiveness of breast cancer cells (Park and Kim, 2015). Herein, we additionally report that the 'LPS-MyD88' cascade acts upstream of BLT2 in ICAM-1 expression by MDA-MB-231 breast cancer cells. The role of BLT2 in this 'LPS-MyD88'-induced ICAM-1 expression was also demonstrated by BLT2 overexpression experiments; we observed that enforced BLT2 expression could enhance the levels of ICAM-1 mRNA and protein (Fig. 2C).

Our results demonstrate that ERK is a downstream component of the MyD88-BLT2 cascade (Fig. 5D). ERK activation has been previously shown to be critical for ICAM-1 expression (Watanabe et al., 2011), and in accordance with that report, we found that LPS-induced ERK activation is critical for ICAM-1 expression in MDA-MB-231 cells and for their adhesion to monocytes (Figs. 5B and 5C), Several reports have suggested that NF-KB lies downstream of ERK (Cargnello and Roux, 2011), thus regulating ICAM-1 expression (Lin et al., 2005; Rui et al., 2015). Similarly, we previously reported that NF-kB lies downstream of the LPS-MyD88-BLT2 cascade in MDA-MB-231 cells (Park and Kim, 2015). We also found that ERK inhibition by PD98059 resulted in a marked reduction in the phosphorylation of $\mathrm{IKB} \alpha$ induced by LPS and that LPS-induced ICAM-1 expression is suppressed by the NF-kB inhibitor Bay 11-7082 (data not shown). These findings suggest that a BLT2-ERK-NF-KB cascade is likely associated with the induction of ICAM-1 expression by LPS.

In summary, our results demonstrate that LPS upregulates ICAM-1 expression in MDA-MB-231 breast cancer cells via a MyD88-BLT2-ERK signaling cascade, thus facilitating their adhesion to monocytes. The elucidation of this mechanism provides significant insight into the progression of breast cancer, especially within the microenvironment of the infected tumor.

\section{ACKNOWLEDGMENTS}

This work was supported by Bio \& Medical Technology Development Program Grant (2012M3A9C5048709, 2012M3A9C1 053532), through the National Research Foundation funded by the Ministry of Science, Information and Communication Technologies (ICT) and Future Planning, Republic of Korea. This work was also supported by a Korea University Grant and BK21 Plus Program (School of Life Sciences and Biotechnology, Korea University).

\section{REFERENCES}

Blot, E., Chen, W., Vasse, M., Paysant, J., Denoyelle, C., Pille, J.Y., Vincent, L., Vannier, J.P., Soria, J., and Soria, C. (2003). Cooperation between monocytes and breast cancer cells promotes factors involved in cancer aggressiveness. $\mathrm{Br} \mathrm{J}$. Cancer 88, 1207-1212.

Cargnello, M., and Roux, P.P. (2011). Activation and function of the MAPKs and their substrates, the MAPK-activated protein kinases. Microbiol. Mol. Biol. Rev. 75, 50-83.

Chaves, M.M., Marques-da-Silva, C., Monteiro, A.P., Canetti, C. and Coutinho-Silva, R. (2014). Leukotriene B4 modulates P2X7 receptor-mediated Leishmania amazonensis elimination in murine macrophages. J. Immunol. 192, 4765-4773.

Chen, C., and Khismatullin, D.B. (2014). Lipopolysaccharide induces the interactions of breast cancer and endothelial cells via activated monocytes. Cancer Lett. 345, 75-84.

Chittezhath, M., Dhillon, M.K., Lim, J.Y., Laoui, D., Shalova, I.N., Teo, Y.L., Chen, J., Kamaraj, R., Raman, L., Lum, J., et al. (2014). Molecular profiling reveals a tumor-promoting phenotype of monocytes and macrophages in human cancer progression. Immunity 41, 815-829.

Coste, I., Le Corf, K., Kfoury, A., Hmitou, I., Druillennec, S., Hainaut, P., Eychene, A., Lebecque, S., and Renno, T. (2010). Dual function of MyD88 in RAS signaling and inflammation, leading to mouse and human cell transformation. J. Clin. Invest. 120, 3663-3667.

Evani, S.J., Prabhu, R.G., Gnanaruban, V., Finol, E.A., and Ramasubramanian, A.K. (2013). Monocytes mediate metastatic breast tumor cell adhesion to endothelium under flow. FASEB J. 27, 3017-3029

Funk, C.D. (2001). Prostaglandins and leukotrienes: advances in eicosanoid biology. Science 294, 1871-1875.

Huang, W.C., Chan, S.T., Yang, T.L., Tzeng, C.C., and Chen, C.C. (2004). Inhibition of ICAM-1 gene expression, monocyte adhesion and cancer cell invasion by targeting IKK complex: molecular and functional study of novel alpha-methylenegamma-butyrolactone derivatives. Carcinogenesis 25, 19251934

Hubbard, A.K., and Rothlein, R. (2000). Intercellular adhesion molecule-1 (ICAM-1) expression and cell signaling cascades. Free Radic. Biol. Med. 28, 1379-1386.

Kim, J.Y., Lee, W.K., Yu, Y.G., and Kim, J.H. (2010). Blockade of LTB4-induced chemotaxis by bioactive molecules interfering with the BLT2-Galphai interaction. Biochem. Pharmacol. 79, 1506-1515.

Kim, H., Choi, J.A., Park, G.S., and Kim, J.H. (2012). BLT2 upregulates interleukin-8 production and promotes the invasiveness of breast cancer cells. PLoS One 7, e49186.

Kim, H., Park, G.S., Lee, J.E., and Kim, J.H. (2013a). A leukotriene B4 receptor-2 is associated with paclitaxel resistance in MCF7/DOX breast cancer cells. Br J. Cancer 109, 351-359.

Kim, J.Y., Kim, H., Jung, B.J., Kim, N.R., Park, J.E., and Chung, D.K (2013b). Lipoteichoic acid isolated from Lactobacillus plantarum suppresses LPS-mediated atherosclerotic plaque inflammation. Mol. Cells 35, 115-124.

Kim, H. Choi, J.A., and Kim, J.H. (2014). Ras promotes transforming growth factor-beta (TGF-beta)-induced epithelialmesenchymal transition via a leukotriene B4 receptor-2-linked cascade in mammary epithelial cells. J. Biol. Chem. 289, 2215122160.

Lee, S.J., Choi, E.K., Seo, K.W., Bae, J.U., Kim, Y.H., Park, S.Y., Oh, S.O., and Kim, C.D. (2013). 5-Lipoxygenase plays a pivotal role in endothelial adhesion of monocytes via an increased expression of Mac-1. Cardiovasc. Res. 99, 724-733.

Lin, F.S., Lin, C.C., Chien, C.S., Luo, S.F., and Yang, C.M. (2005). Involvement of p42/p44 MAPK, JNK, and NF-kappaB in IL1beta-induced ICAM-1 expression in human pulmonary epithelial cells. J. Cell Physiol. 202, 464-473.

Mantovani, A., Allavena, P., Sica, A., and Balkwill, F. (2008). Cancerrelated inflammation. Nature 454, 436-444.

Park, G.S., and Kim, J.H. (2015). Myeloid differentiation primary response gene 88-leukotriene B4 receptor 2 cascade mediates lipopolysaccharide-potentiated invasiveness of breast cancer cells. Oncotarget 6, 5749-5759. 
Powell, W.S., Gravel, S., Khanapure, S.P., and Rokach, J. (1999). Biological inactivation of 5-oxo-6,8,11,14-eicosatetraenoic acid by human platelets. Blood 93, 1086-1096.

Rakoff-Nahoum, S., and Medzhitov, R. (2009). Toll-like receptors and cancer. Nat. Rev. Cancer 9, 57-63.

Rosette, C., Roth, R.B., Oeth, P., Braun, A., Kammerer, S., Ekblom, J., and Denissenko, M.F. (2005). Role of ICAM1 in invasion of human breast cancer cells. Carcinogenesis 26, 943-950.

Rui, W., Guan, L., Zhang, F., Zhang, W., and Ding, W. (2015). PM 2.5-induced oxidative stress increases adhesion molecules expression in human endothelial cells through the ERK/AKT/NFkappaB-dependent pathway. J. Appl. Toxicol. doi: 10.1002/jat.3143. [Epub ahead of print]

Schroder, C., Witzel, I., Muller, V., Krenkel, S., Wirtz, R.M., Janicke, F., Schumacher, U., and Milde-Langosch, K. (2011). Prognostic value of intercellular adhesion molecule (ICAM)-1 expression in breast cancer. J. Cancer Res. Clin. Oncol. 137, 1193-1201.

Seo, J.M., Cho, K.J., Kim, E.Y., Choi, M.H., Chung, B.C., and Kim, J.H. (2011). Up-regulation of BLT2 is critical for the survival of bladder cancer cells. Exp. Mol. Med. 43, 129-137.

Seo, J.M., Park, S., and Kim, J.H. (2012). Leukotriene B4 receptor2 promotes invasiveness and metastasis of ovarian cancer cells through signal transducer and activator of transcription 3 (STAT3)-dependent up-regulation of matrix metalloproteinase 2. J. Biol. Chem. 287, 13840-13849.

Silverman, M.D., Zamora, D.O., Pan, Y., Texeira, P.V., Planck, S.R. and Rosenbaum, J.T. (2001). Cell adhesion molecule expression in cultured human iris endothelial cells. Invest Ophthalmol. Vis. Sci. 42, 2861-2866.

Usami, Y., Ishida, K., Sato, S., Kishino, M., Kiryu, M., Ogawa, Y., Okura, M., Fukuda, Y., and Toyosawa, S. (2013). Intercellular adhesion molecule-1 (ICAM-1) expression correlates with oral cancer progression and induces macrophage/cancer cell adhesion. Int. J. Cancer 133, 568-578.

van de Stolpe, A., and van der Saag, P.T. (1996). Intercellular adhesion molecule-1. J. Mol. Med. 74, 13-33.

Watanabe, N., Shikata, K., Shikata, Y., Sarai, K., Omori, K., Kodera R., Sato, C., Wada, J., and Makino, H. (2011). Involvement of MAPKs in ICAM-1 expression in glomerular endothelial cells in diabetic nephropathy. Acta Med. Okayama 65, 247-257.

Zhu, X.W., and Gong, J.P. (2013). Expression and role of icam-1 in the occurrence and development of hepatocellular carcinoma. Asian Pac. J. Cancer Prev. 14, 1579-1583. 